Article

\title{
Fluorescence Emission Behaviors of the L-Cysteine/Au(I) Complex in a Cyclodextrin-Based Metal-Organic Framework
}

\author{
Wataru Michida ${ }^{1}$, Anna Nagai ${ }^{1}$, Mina Sakuragi ${ }^{1}$, Tadashi Okobira ${ }^{2}$ and Katsuki Kusakabe ${ }^{1, *}$ \\ 1 Department of Nanoscience, Sojo University, 4-22-1 Ikeda, Nishi-ku, Kumamoto 860-0082, Japan; \\ michida1005@icloud.com (W.M.); g1814D01@m.sojo-u.ac.jp (A.N.); d08b0101@nano.sojo-u.ac.jp (M.S.) \\ 2 Department of Creative Engineering, National Institute of Technology, Ariake College, Omuta-shi, \\ Fukuoka 836-8585, Japan; okobira@ariake-nct.ac.jp \\ * Correspondence: kusakabe@nano.sojo-u.ac.jp
}

Received: 4 November 2020; Accepted: 25 November 2020; Published: 27 November 2020

\begin{abstract}
Aggregation-induced emission (AIE) molecules are nonemissive in dilute solution but emit intensely upon aggregation in complete contrast to aggregation-caused quenching (ACQ) molecules. The emission of ACQ molecules, such as fluorescein, that have been encapsulated into the hydrophilic nanopores in a cyclodextrin-based metal-organic framework (CD-MOF) was reported to be enhanced due to the disappearance of concentration quenching and the restriction of thermal motion. However, the contribution of the restriction of thermal motion in CD-MOF could not be elucidated. In this study, an AIE-active L-cysteine/Au(I) (L-Cys/Au(I)) complex was synthesized and introduced into the nanopores of $\mathrm{CD}-\mathrm{MOF}$ via a co-crystallization method. We determined the amount and chemical composition of the L-Cys/Au(I) complex in CD-MOF. The fluorescence intensity of the $\mathrm{L}-\mathrm{Cys} / \mathrm{Au}(\mathrm{I}) @ \mathrm{CD}-\mathrm{MOF}$ composite was investigated. The L-Cys/Au(I) complex that was synthesized from $\mathrm{Au}(\mathrm{III})$ chloride and L-cysteine was found to be a linear oligomer consisting of $\mathrm{Cys}_{5} \mathrm{Au}_{4}$. For the L-Cys/Au(I)@CD-MOF composite with a L-Cys/Au(I) complex of 0.45 per hydrophilic nanopore, the total fluorescence intensity of the isolated L-Cys/Au(I) complex in CD-MOF exceeded that of the L-Cys/Au(I) complex in the solid-state due to the restriction of the thermal motion without the aggregation of the complex.
\end{abstract}

Keywords: porous material; crystal; photoluminescence; aggregation-induced emission (AIE)

\section{Introduction}

Aggregation-induced emission (AIE) is a unique photophysical phenomenon that is completely opposed to the aggregation-caused quenching (ACQ) effect observed in typical luminophore systems. The AIE fluorogens are non-luminescent in a dilute solution but emit intensely upon aggregation formation. Their aggregates show strong fluorescence mainly due to the restriction of their thermal motion in the aggregate state. However, the AIE mechanism in terms of luminescent chromosphere is still not clear [1]. L-Cysteine/Au(I) (L-Cys/Au(I)) complexes are synthesized at room temperature using cysteine as both a reducing agent and capping ligand [2]. $\mathrm{Au}(\mathrm{III})$ is reduced to $\mathrm{Au}(\mathrm{I})$ with $\mathrm{L}$-cysteine and then $\mathrm{Au}(\mathrm{I})$ stabilizes with excess L-cysteine to form a L-Cys/Au(I) complex that is nonluminescent in an aqueous solution [3]. In recent years, it was shown that an Au(I)-thiolate complex with fluorescent properties in a poor solvent or solid-state can be an interesting molecular luminescent probe for biological sensing applications [4,5]. Wang et al. [6] reported the doping of a polyelectrolyte-Au nanocluster (Au NC) complex into silica particles for biolabeling and bioimaging. The emission intensity of the Au NC-doped silica particles was increased due to the rigidifying effect 
of the silica matrix. Zhao et al. [7] presented a novel design of core-shell $\mathrm{Au}(\mathrm{I})$-glutathione@ $@ \mathrm{SiO}_{2}$ nanoparticles. Metal cations that are smaller than the ring size of silica network structure can penetrate through the shell light up the glutathione/Au(I) complex via the AIE mechanism. If an L-Cys/Au(I) complex with AIE properties is introduced into a nanospace surrounded by an optically transparent material, then the thermal motion of a single complex can be restricted, thereby resulting in the AIE phenomenon without aggregation.

The cyclodextrin-based metal-organic framework (CD-MOF) is crystallized via the complex formation of $\gamma$-cyclodextrin $(\gamma-\mathrm{CD})$ and alkali metal ion. CD-MOF, which is a porous organic crystal, has a body-centered cubic structure of $(\gamma-C D)_{6}$ units that consists of six $\gamma$-CDs and is characterized by a high specific surface area, high light transmission, and few defects [8,9]. As shown in Figure 1 , hydrophilic pores $(1.7 \mathrm{~nm})$ with $\mathrm{OH}$ groups inside was formed at the center of $(\gamma-\mathrm{CD})_{6}$. The inside of the barrel-shaped nanopore $(1 \mathrm{~nm})$ that was formed by a pair of $\gamma$-CDs is hydrophobic. The hydrophobic nanopores are linked to the hydrophilic nanopores to form a pore structure. Michida et al. [10] reported the immobilization of typical fluorescent dyes, namely fluorescein (Flu) and rhodamine B (Rh B), into CD-MOF crystals. Hydrophilic Flu and Rh B were present in the spherical nanopores at the center of the $(\gamma-C D)_{6}$ unit. Isolated Flu and Rh B molecules emitted strong yellow and red fluorescence, respectively, in the solid-state due to the disappearance of concentration quenching and the restriction of thermal motion. If AIE-active molecules can be introduced into the CD-MOF, the fluorescence intensity will increase due to the suppression of thermal motion, without considering the effects of concentration quenching.

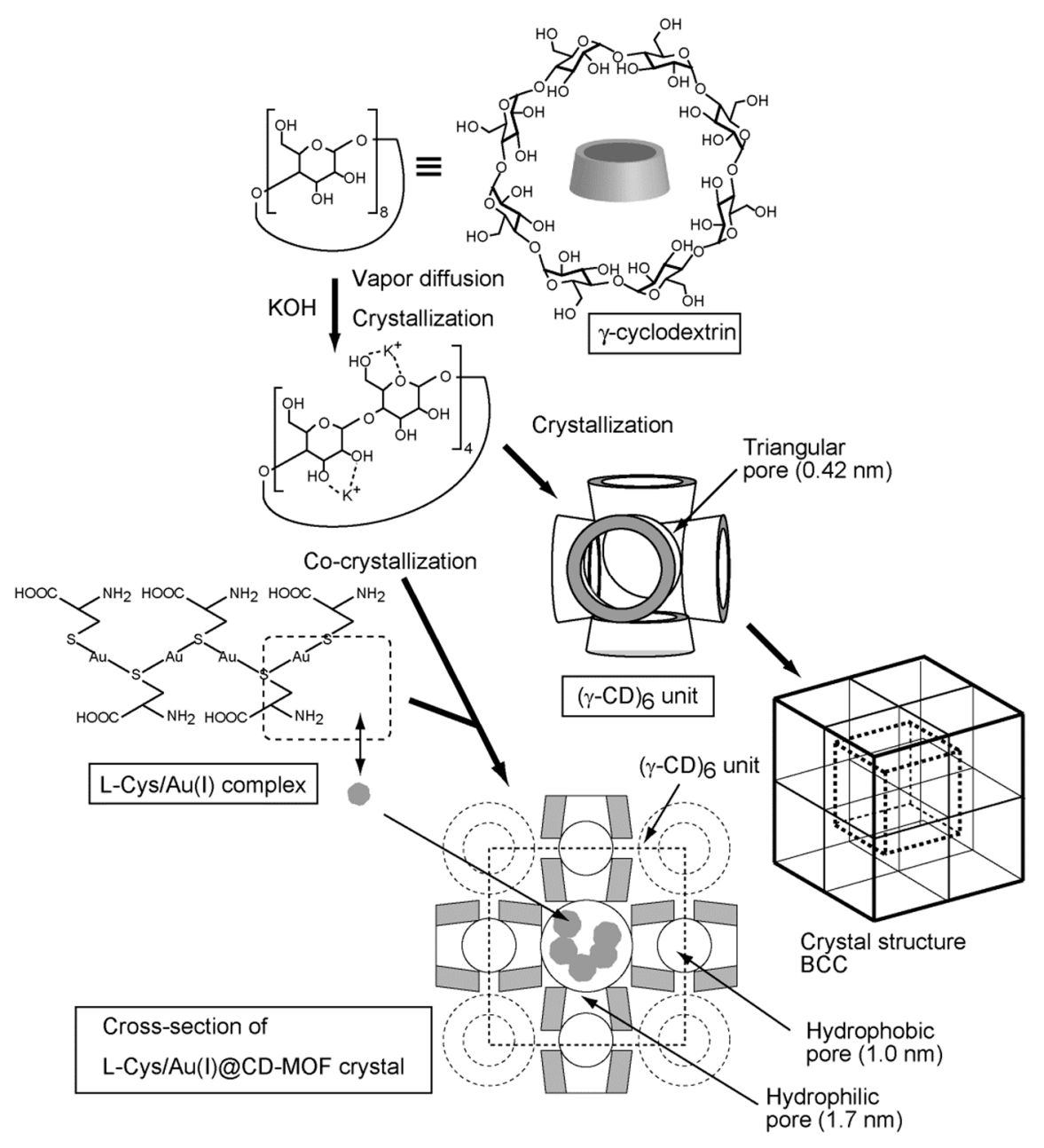

Figure 1. Schematic of the synthesis of L-Cys/Au(I)@CD-MOF. 
In this study, tetrachloroauric(III) acid tetrahydrate is used as a precursor, and L-cysteine is used to both cap the ligand and reduce the agent that produces the $\mathrm{L}-\mathrm{Cys} / \mathrm{Au}(\mathrm{I})$ complex. The introduction of the L-Cys/Au(I) complex into the CD-MOF crystal was conducted via the co-crystallization method using methanol vapor diffusion. The crystallization yield, crystal structure, and porosity properties of the obtained L-Cys/Au(I)@CD-MOF crystal and the amount of the complex in CD-MOF were characterized. The relationship between the fluorescence intensity and the molecular number of $\mathrm{L}-\mathrm{Cys} / \mathrm{Au}(\mathrm{I})$ complex per $(\gamma-\mathrm{CD})_{6}$ unit was investigated.

\section{Experimental Design}

\subsection{Materials}

L-Cysteine (L-Cys), tetrachloroauric(III) acid tetrahydrate $\left(\mathrm{HAuCl}_{4} \cdot \mathrm{H}_{2} \mathrm{O}\right)$, potassium hydroxide $(\mathrm{KOH})$, methanol, and dichloromethane were purchased from FUJIFILM Wako Pure Chemical Corporation (Osaka, Japan). $\gamma$-Cyclodextrin was obtained from Tokyo Chemical Industry (TCI) (Tokyo, Japan). All other reagents were of analytical grade and used without further purification.

\subsection{Synthesis of L-Cys/Au(I) Complex}

An L-cysteine solution $(100 \mathrm{mmol} / \mathrm{L}, 8 \mathrm{~mL})$ was added dropwise to an $\mathrm{HAuCl}_{4}$ solution $(4 \mathrm{mmol} / \mathrm{L}$, $50 \mathrm{~mL}$ ) while stirring. The mixed solution was continuously stirred for $1 \mathrm{~h}$ to form the L-Cys/Au(I) complex. After the reaction, the white precipitate was collected by centrifugation ( $3500 \mathrm{rpm}$ for $10 \mathrm{~min}$ ) and washed with ion-exchanged water. This operation was repeated twice to completely remove impurities. The obtained precipitate was dried under a vacuum overnight.

\subsection{Crystallization of $C D-M O F$ and $L-C y s / A u(I) @ C D-M O F$}

CD-MOF was prepared via the methanol vapor diffusion method at room temperature [8]. $\gamma$-CD $(163 \mathrm{mg})$ was dissolved in a $\mathrm{KOH}$ solution $(200 \mathrm{mmol} / \mathrm{L}, 5 \mathrm{~mL})$. To introduce the L-Cys/Au(I) complex into the CD-MOF crystal, the co-crystallization method [9] was applied. $\gamma$-CD (163 mg) and the $\mathrm{L}-\mathrm{Cys} / \mathrm{Au}(\mathrm{I})$ complex $(20 \mathrm{mg}$ as a standard weight) were dissolved in the $\mathrm{KOH}$ solution $(200 \mathrm{mmol} / \mathrm{L}$, $5 \mathrm{~mL})$. The $\gamma$-CD solution with and without the L-Cys/Au(I) complex was poured into three glass petri dishes (diameter $5 \mathrm{~cm}$ and depth $1.5 \mathrm{~cm}$ ) and contact with methanol vapor in a closed system for 3 days to crystallize CD-MOF. To investigate the effect of the loading of L-Cys/Au(I) complex in CD-MOF on the fluorescent intensity, the co-crystallization was performed by adjusting the amount of L-Cys/Au(I) complex from 5 to $50 \mathrm{mg}$ without changing the other conditions. The obtained crystals were washed with methanol, soaked in dichloromethane for 3 days and dried under vacuum overnight at $45^{\circ} \mathrm{C}$.

\subsection{Characterization}

The crystal structure of CD-MOF was confirmed using a powder X-ray diffraction (XRD) method (PANalytical, XRD X'Pert PRO, $\mathrm{Cu}-\mathrm{K} \alpha$ radiation). The BET surface area of L-Cys/Au(I)@CD-MOF was assessed via multipoint $\mathrm{N}_{2}$ adsorption-desorption experiments which were performed on an automatic specific surface area and pore distribution analyzer (Shimadzu TriStar II, Kyoto, Japan). The sample was outgassed at $150{ }^{\circ} \mathrm{C}$ for $6 \mathrm{~h}$ in vacuum before adsorption measurement. The specific surface area was calculated via the Brunauer-Emmett-Teller (BET) method. Scanning electron microscope (SEM) images of the crystals were captured and an element mapping was obtained using EDS (energy dispersive spectroscopy) via SEM-EDS (VE-8800, Keyence Corp., Osaka, Japan). The formation of the L-Cys/Au(I) complex was investigated using ATR-FT-IR analysis (Spotlight 200, Perkin Elmer, MA, USA). The molar ratio of L-Cys to $\mathrm{Au}$ in the L-Cys/Au(I) complex was evaluated via TG analysis (TG/DTA 7220, Hitachi High-Tech Sci. Corp., Tokyo, Japan). Thermogravimetric (TG) curves were recorded at a heating rate of $2{ }^{\circ} \mathrm{C} \cdot \mathrm{min}^{-1}$ from room temperature to $550{ }^{\circ} \mathrm{C}$ under a flow of air. The concentration of $\mathrm{L}-\mathrm{Cys} / \mathrm{Au}(\mathrm{I})$ complex that was dissolved in the $\mathrm{KOH}$ solution was measured with a UV-vis spectrophotometer (H550, JASCO Corp, Tokyo, Japan). The fluorescence emission of L-Cys/Au(I) in 
CD-MOF was observed with confocal laser scanning micrographs (CLSM) (LSM 510 META, Carl Zeiss AG, Germany). Fluorescent spectra of L-Cys/Au(I)@CD-MOF were recorded on a fluorescence spectrometer (Shimadzu, RF-6000, Kyoto, Japan). An excitation slit width of $5 \mathrm{~nm}$ and an emission slit width of $10 \mathrm{~nm}$ were used to record the fluorescence spectra in the wavelength range 400-600 nm. The mass of L-Cys/Au(I) in CD-MOF was analyzed via matrix-assisted laser desorption/ionization time-of-flight mass spectrometry (MALDI-TOF MS, Bruker Corp., MA, USA) using a spectrometer that was equipped with a nitrogen laser that operated at $337 \mathrm{~nm}$. $\gamma$-Cyano-4-hydroxycinnamic acid was used as a matrix for the MALDI-TOF MS analysis. The measurement was conducted in reflection mode at an accelerating voltage of $20 \mathrm{kV}$.

\section{Results and Discussion}

\subsection{Characterization of the L-Cys/Au(I) Complex}

The reduction of $\mathrm{HAuCl}_{4}$ in the presence of L-cysteine has been widely employed for the synthesis of the L-Cys/Au(I) complex [3,11]. $\mathrm{Au}(\mathrm{III})$ was found to be reduced to $\mathrm{Au}(\mathrm{I})$ due to the formation of disulfide cystine from L-cysteine. $\mathrm{Au}(\mathrm{I})$ can be stabilized with excess L-cysteine to form L-Cys/Au(I) complex. The reaction yield of the L-Cys/Au(I) complex was 39.5\%. As shown in Figure 2, the L-Cys/Au(I) complex emitted red fluorescence when illuminated with black light (wavelength $365 \mathrm{~nm}$ ). FT-IR spectra of L-cysteine and the L-Cys/Au(I) complex are shown in Figure 3. The absorption band at $2552 \mathrm{~cm}^{-1}$ can be assigned to the SH stretching vibration of L-cysteine but disappeared upon the complexation of L-cysteine with $\mathrm{Au}(\mathrm{I})$. Thermogravimetric analysis of the $\mathrm{L}-\mathrm{Cys} / \mathrm{Au}(\mathrm{I})$ complex was conducted from room temperature to $550^{\circ} \mathrm{C}$. As shown in Figure 4, oxidation of L-cysteine occurred mainly at $180-250^{\circ} \mathrm{C}$, and the mass was constant at $500{ }^{\circ} \mathrm{C}$. The molar ratio of L-cysteine to Au was approximately 1.1, under the assumption of that metallic Au remained at $500^{\circ} \mathrm{C}$. The L-Cys/Au(I) complex was analyzed via MALDI-TOF MS, and a signal with $\left(\mathrm{Cys}_{5} \mathrm{Au}_{4}+2 \mathrm{~K}\right)^{++}$at $1490.4 \mathrm{~m} / \mathrm{z}$ was detected. As a result, the structure of the synthesized L-Cys/Au(I) complex is posited to correspond to (Cys-Au(I)) $)_{4}$-Cys. Guo et al. [12] prepared an L-Cys/Au(I) complex for $\mathrm{Ca}^{2+}$ detection based on its AIE property and characterized it via MALDI-TOF MS. The main composition of the products was $\left(\mathrm{Cys}_{7} \mathrm{Au}_{6}\right)^{+}$.
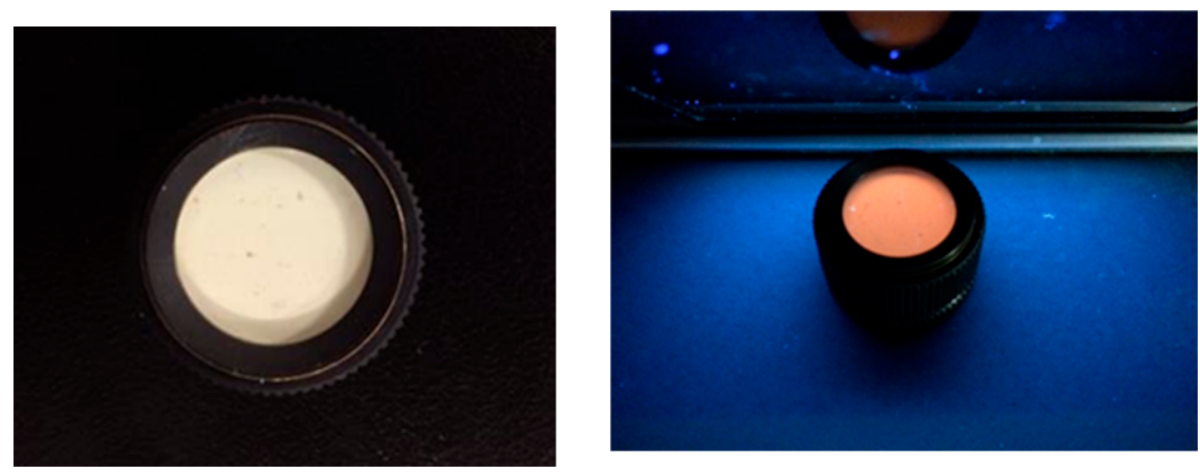

Figure 2. Image of the L-Cys/Au(I) complex without (left) and with (right) black light irradiation (wavelength $365 \mathrm{~nm}$ ). 


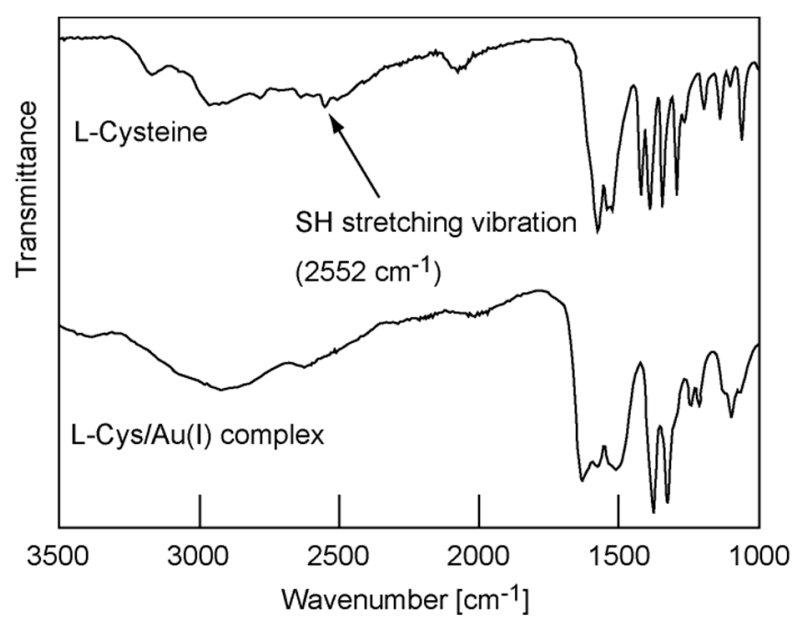

Figure 3. IR spectra of L-Cysteine and the L-Cys/Au(I) complex.

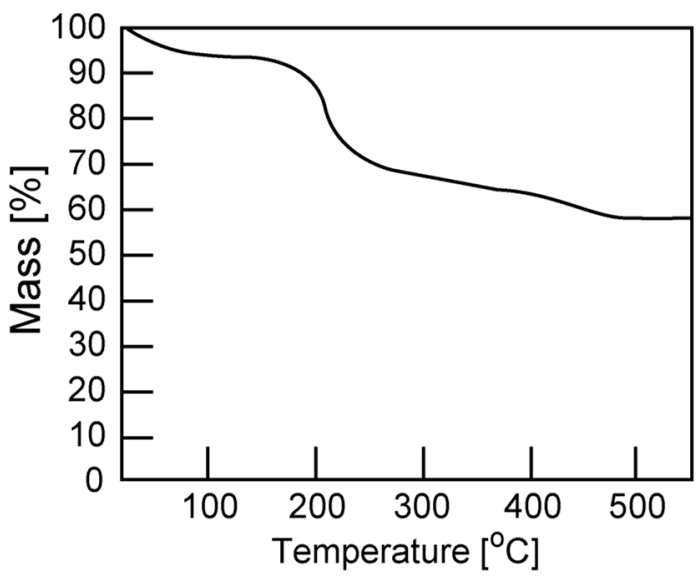

Figure 4. Thermogravimetric analysis of the L-Cys/Au(I) complex.

\subsection{Characterization of $L-C y s / A u(I) @ C D-M O F$}

Encapsulation of the L-cysteine/Au(I) complex into CD-MOF was conducted via the co-crystallization method. The synthesized L-Cys/Au(I)@CD-MOF was a light yellow powder and emitted red fluorescence under black light irradiation (wavelength $365 \mathrm{~nm}$ ). From the consistency of the X-ray patterns of the L-Cys/Au(I)@CD-MOF and CD-MOF in Figure 5, it was demonstrated that the crystal structure was maintained. However, the crystallinity of L-Cys/Au(I)@CD-MOF was low compared to that of CD-MOF due to the presence of the complex in the nanopores. To evaluate the dispersion of the L-Cys/Au(I) complex in CD-MOF, three-dimensional mapping of the fluorescence intensity was conducted using confocal laser scanning micrographs. As shown in Figure 6, fluorescence was observed almost uniformly from the inside of the cross section of the CD-MOF structure.

L-Cys/Au(I)@CD-MOF was dissolved in a $\mathrm{KOH}$ solution and then the dissolved L-Cys/Au(I) complex concentration was determined by UV-vis spectrophotometry, according to which $8 \mathrm{wt} \%$ of the L-Cys/Au(I) complex was introduced into CD-MOF under normal conditions, as discussed in Section 2.2. MALDI-TOF-MS spectra from L-Cys/Au(I)@CD-MOF were compared to spectra of $\mathrm{L}-\mathrm{Cys} / \mathrm{Au}(\mathrm{I})$ complex. The peak of $\left(\mathrm{Cys}_{5} \mathrm{Au}_{4}+2 \mathrm{~K}\right)^{++}$at $1490.4 \mathrm{~m} / \mathrm{z}$ was also detected in the MALDI-TOF MS spectra of L-Cys/Au(I)@CD-MOF. Additional signals of $\left(\mathrm{Cys}_{6} \mathrm{Au}(\mathrm{I})_{6}\right)$ and $\left(\mathrm{Au}_{13}\right)$ were detected at approximately $1920 \mathrm{~m} / \mathrm{z}$ and $2568 \mathrm{~m} / \mathrm{z}$, respectively. From these results, it was found that five to six $\mathrm{L}-\mathrm{Cys} / \mathrm{Au}(\mathrm{I})$ units were present in a hydrophilic nanopore in CD-MOF. 


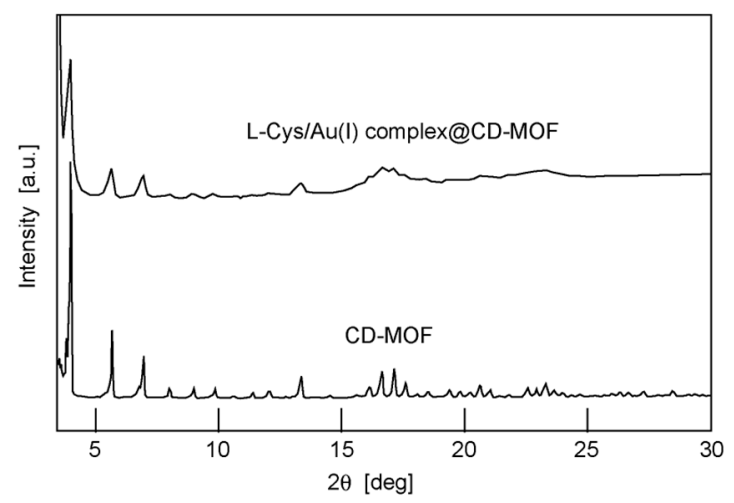

Figure 5. X-ray diffraction (XRD) patterns of CD-MOF and L-Cys/Au(I)@CD-MOF.
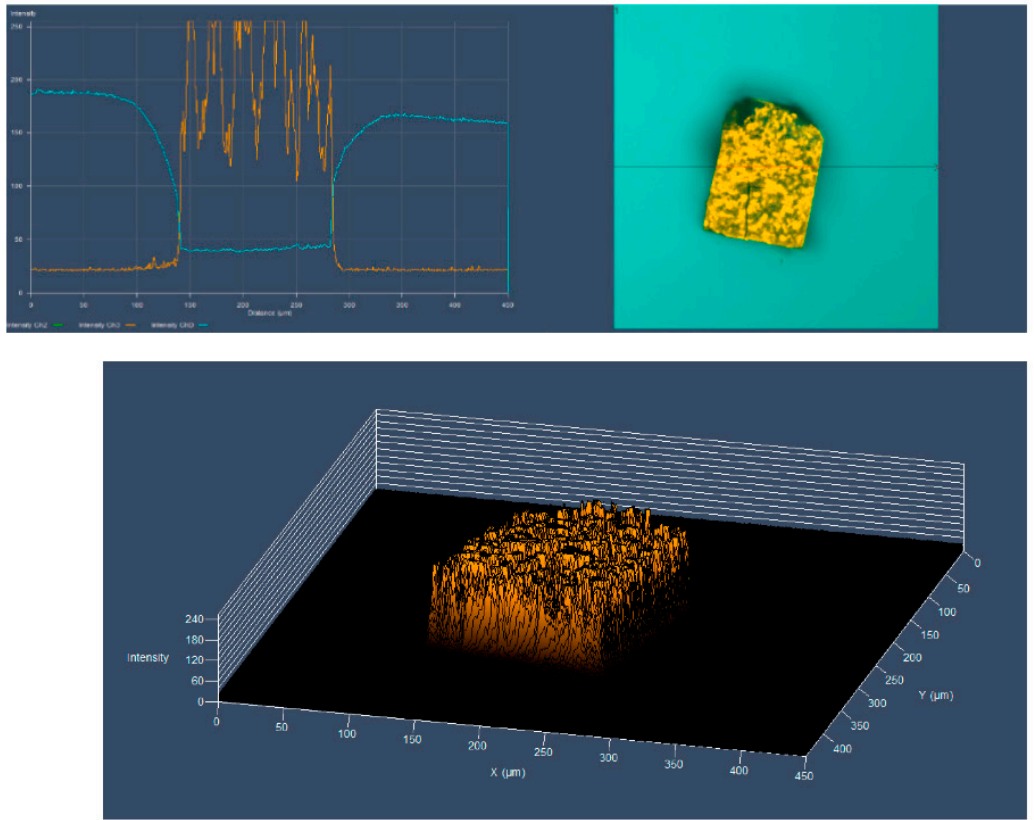

Figure 6. Representative CLSM image of L-Cys/Au(I)@CD-MOF. (Upper right) An optical image of L-Cys/Au(I)@CD-MOF (upper left), the one-dimensional of fluorescence intensity, and (bottom) a $3 \mathrm{D}$ reconstruction.

\subsection{Fluorescence Properties of L-Cys/Au(I)@CD-MOF}

To detect the aggregation-induced emission (AIE) of the L-Cys/Au(I) complex, we compared the solid-state fluorescence intensities of the L-Cys/Au(I) complex and L-Cys/Au(I)@CD-MOF. The L-Cys/Au(I) complex was diluted with $\mathrm{KBr}$ to a concentration of $8 \mathrm{wt} \%$. Figure 7 shows that a maximum emission wavelength of $668 \mathrm{~nm}$ was obtained in both samples with excitation wavelength of $406 \mathrm{~nm}$. The fluorescence intensity was increased approximately 10 times by introducing the L-Cys/Au(I) complex into CD-MOF. The fluorescence intensity of the pure L-Cys/Au(I) complex was almost the same as that of the complex with a concentration of $8 \mathrm{wt} \%$.

The L-cys/Au(I) complex is known to be an AIE phosphor. Therefore, fluorescence emission was observed in the solid-state, as shown in Figure 7. This is explained by the reduction in the intramolecular rotation of the L-Cys/Au(I) complex. However, it was found that the fluorescence intensity was significantly increased by introducing an aggregate of five or six units of L-Cys-/Au(I) into a hydrophilic nanopore of CD-MOF. The L-Cys/Au(I) complex that is confined in CD-MOF further suppresses the molecular mobility due to the strong hydrogen bond with the 6-OH group in the hydrophilic nanopore. The CD-MOF is transparent to light in the visible region. The L-Cys/Au(I) 
complex is isolated and highly dispersed in CD-MOF. As a result, the fluorescence intensity of L-Cys/Au(I)@CD-MOF was enhanced by the high transmission of the excitation light, the suppression of the interaction of the internal fluorescent molecules, and the reduced reabsorption of the light that was emitted by the fluorescent molecules.

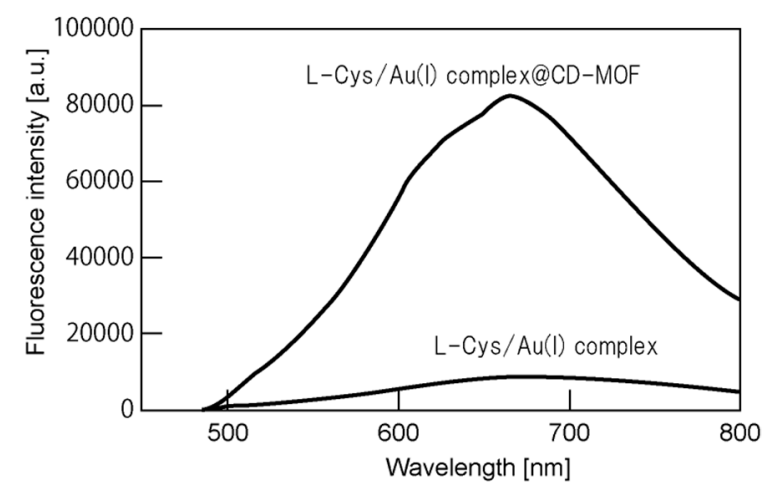

Figure 7. Emission spectra of the L-Cys/Au(I) complex and L-Cys/Au(I)@CD-MOF with the same concentration of L-Cys/Au(I).

If the L-Cys ${ }_{5} \mathrm{Au}_{4}$ complex (apparent molecular mass $=1389.0$ ) is introduced into the hydrophilic nanopores in $\mathrm{CD}-\mathrm{MOF}$ under the standard experimental condition, then the mass concentration of L-Cys ${ }_{5} \mathrm{Au}_{4}$ complex with respect to CD-MOF becomes $8 \mathrm{wt} \%$, which corresponds to 0.45 in term $\mathrm{s}$ of the molecular number of the $\mathrm{L}-\mathrm{Cys}_{5} \mathrm{Au}_{4}$ complex per $(\gamma-\mathrm{CD})_{6}$ unit. Thus, $45 \%$ of all hydrophilic nanopores in CD-MOF are occupied by the L-Cys/Au(I) complex, and the remaining $55 \%$ are voids. To investigate the effect of the molecular number of L-Cys $\mathrm{Au}_{4}$ complex per $(\gamma-\mathrm{CD})_{6}$ unit on the fluorescent intensity, we prepared L-Cys/Au(I)@CD-MOF with various concentrations (the molecular number of the L-Cys $\mathrm{Au}_{4}$ complex per $\left.(\gamma-\mathrm{CD})_{6}=0.20-0.92\right)$. Emission spectra of the $\mathrm{L}-\mathrm{Cys} / \mathrm{Au}(\mathrm{I}) @ \mathrm{CD}-\mathrm{MOF}$ with various amounts of the L-Cys/Au(I) complex are shown in Figure 8 . The $\mathrm{N}_{2}$ adsorption-desorption curves of CD-MOF and L-Cys/Au(I)@CD-MOF displayed the type I isotherm, indicating microporous solid and chemisorption. In the BET specific surface area measurement, the surface areas of the complexes were in the range of $900-700 \mathrm{~m}^{2} / \mathrm{g}$, and the BET specific surface area of L-Cys/Au(I)@CD-MOF tended to decrease apparently due to the increase in mass of the L-Cys $/ \mathrm{Au}(\mathrm{I})$ complex in CD-MOF. Figure 9 shows the relationship between the molecular number of the L-Cys $\mathrm{Au}_{4}$ complex per $(\gamma-\mathrm{CD})_{6}$ unit and the fluorescence intensity. When the molecular number of the L-Cys ${ }_{5} \mathrm{Au}_{4}$ complex per $(\gamma-\mathrm{CD})_{6}$ unit was less than 0.5 , the fluorescence intensity was approximately proportional to the molecular number of the introduced L-Cys/Au(I) complex. This is simply due to the increase in the number of luminescent complexes. When the molecular number was further increased, the fluorescence intensity increased slightly and reached a maximum at a molecular number of 0.8. When a similar fluorescence experiment was conducted using CD-MOF that was encapsulated with fluorescein [10], the fluorescence intensity decreased with increasing fluorescein concentration. This may be due to the attenuation of excitation light through the crystal. Therefore, the saturation of the fluorescence intensity in L-CysAu(I)@CD-MOF is offset by the increase in the number of fluorescent molecules and the decrease in the excitation light. A high fluorescence intensity was maintained even when $92 \%$ of the hydrophilic nanopores were occupied by the complex. 


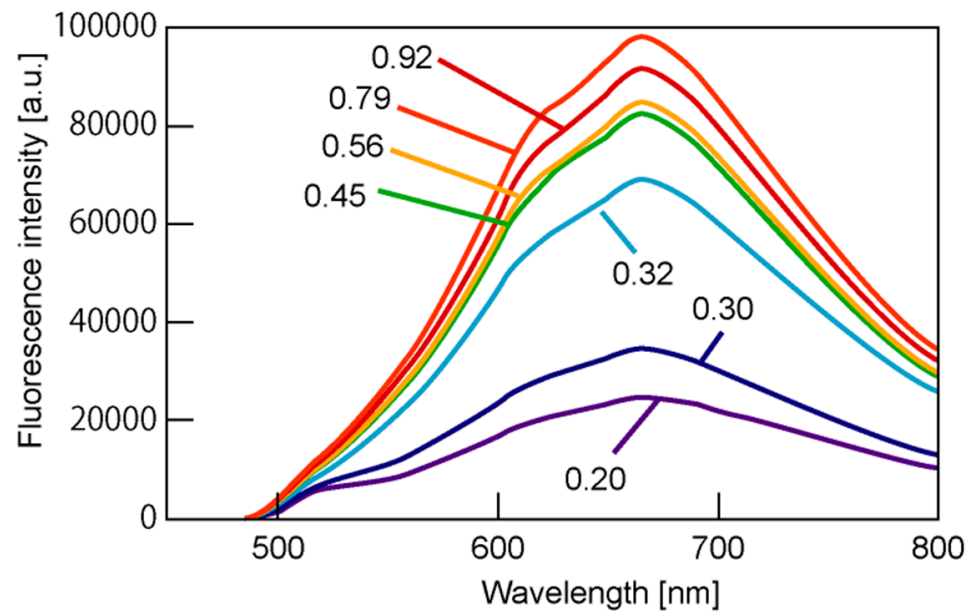

Figure 8. Emission spectra of L-Cys/Au(I)@CD-MOF with various introduction amounts of the $\mathrm{L}-\mathrm{Cys} / \mathrm{Au}(\mathrm{I})$ complex. The numerical values indicate the number of L-Cys/Au(I) complexes per $(\gamma-\mathrm{CD})_{6}$ unit.

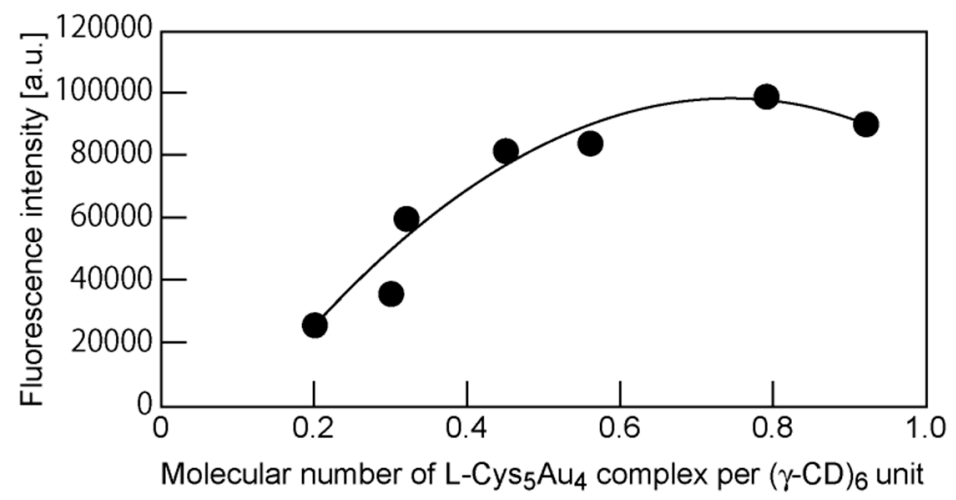

Figure 9. Relationship between the fluorescence intensity of L-Cys/Au(I)@CD-MOF and the number of $\mathrm{L}-\mathrm{Cys} / \mathrm{Au}(\mathrm{I})$ complexes per $(\gamma-\mathrm{CD})_{6}$ unit. (wavelength $=668 \mathrm{~nm}$ ).

\section{Conclusions}

The L-Cys/Au(I) complex that was synthesized from $\mathrm{Au}(\mathrm{III})$ chloride and L-cysteine as both the capping ligand and reducing agent was found to be a linear oligomer consisted of $\mathrm{Cys}_{5} \mathrm{Au}_{4}$. When this yellow L-Cys/Au(I) complex was irradiated with black light (wavelength $365 \mathrm{~nm}$ ), it emitted red fluorescence in a solid-state, thereby demonstrating its aggregation-induced emission.

$\mathrm{L}-\mathrm{Cys} / \mathrm{Au}(\mathrm{I})$ complex was homogeneously encapsulated into CD-MOF by the co-crystallization method and was isolated in a hydrophilic nanopore. The mass concentration of the L-Cys/Au(I) complex in the L-Cys/Au(I)@CD-MOF that was prepared under normal experimental conditions was as high as $8 \mathrm{wt} \%$. Assuming an L-Cys ${ }_{5} \mathrm{Au}_{4}$ oligomer complex with a molecular weight of 1389 , the number of L-Cys/Au(I) complex per hydrophilic nanopore is 0.45 , and the complex is isolated in the CD-MOF. The fluorescence intensity of L-Cys/Au(I)@CD-MOF was 10 times higher than that of the powder that was obtained by diluting the same amount of L-Cys/Au(I) complex with KBr. The AIE-active $\mathrm{L}-\mathrm{Cys} / \mathrm{Au}(\mathrm{I})$ complex in the hydrophilic nanopores showed strong luminescence by suppressing the motion of isolated molecules, regardless of concentration quenching. The relationship between the molecular number of the L-Cys ${ }_{5} \mathrm{Au}_{4}$ complex per $(\gamma-\mathrm{CD})_{6}$ unit and the fluorescence intensity was investigated. When the molecular number of the L-Cys $\mathrm{Au}_{4}$ complex per $(\gamma-\mathrm{CD})_{6}$ unit was less than 0.5 , the fluorescence intensity increased with increasing the molecular number of the introduced $\mathrm{L}-\mathrm{Cys} / \mathrm{Au}(\mathrm{I})$ complex. When the molecular number was further increased, the fluorescence intensity 
increased slightly and reached a maximum at the molecular number of 0.8. A high fluorescence intensity was maintained even when $92 \%$ of the hydrophilic nanopores were occupied by the complex.

Author Contributions: W.M. and K.K. conceived and designed the experiments; W.M., A.N. and M.S. performed the experiments; T.O. and K.K. analyzed the data; K.K. wrote the paper. All authors have read and agreed to the published version of the manuscript.

Funding: This work was supported by a Grant-in-Aid for Exploratory Research (JP15K14217), Scientific Research (B) (JP16H04561, JP19H02508) and a JSPS Research Fellowship (JP16J11710) from JSPS/MEXT Japan. The synchrotron radiation experiments were conducted at SPring-8 BL40B2 (No. 2018A1671).

Acknowledgments: We thank N. Ohta and H. Sekiguchi for experimental help at SPring-8.

Conflicts of Interest: The authors declare no conflict of interest.

\section{References}

1. Chen, Y.; Lam, J.W.Y.; Kwouk, R.T.K.; Tang, B.Z. Aggregation-induced emission: Fundamental understanding and future developments. Mat. Horiz. 2019, 6, 428-433. [CrossRef]

2. Lin, X.; Liu, J.; Tian, M.; Bao, Y.; Shu, T.; Su, L.; Zhang, X. An Aggregation-Induced Phosphorescence-Active "Turn-Off" Nanosensor Based on Ferric-Specific Quenching of Luminescent and Water-Soluble Au(I)-Cystein Nanocomplexes. Anal. Chem. 2020, 92, 6785-6791.

3. Smith, W.E.; Reglinski, J. Gold drugs used in the treatment of rheumatoid arthritis. Perspect. Bioinorg. Chem. 1991, 1, 183-208.

4. He, X.M.; Yam, V.W.-W. Luminescent gold(I) complexes for chemosensing. Coord. Chem. Rev. 2011, 255, 2111-2123.

5. He, X.M.; Chen, E.C.C.; Zhu, N.Y.; Yam, V.W.-W. Selective ion probe for $\mathrm{Mg}^{2+}$ based on $\mathrm{Au}(\mathrm{I}) \cdots \mathrm{Au}(\mathrm{I})$ interactions in a tripodal alkynylgold(I) complex with oligoether pendants. Chem. Commun. 2009, 27, 4016-4018. [CrossRef] [PubMed]

6. Wang, H.; Huang, Z.; Guo, Z.; Yang, W. Polyelectrolyte-assisted preparation of gold nanocluster-doped silica particles with high incorporation efficiency and improved stability. J. Nanopart. Res. 2017, 19, 1-9. [CrossRef]

7. Zhao, T.; Goswami, N.; Li, J.; Yao, Q.; Zhang, Y.; Wang, J.; Zhao, D.; Xie, J. Probing the Microporous structure

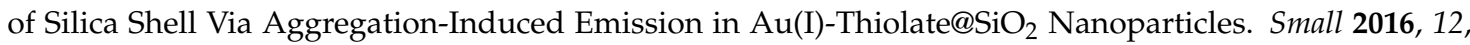
6537-6541. [CrossRef]

8. Smaldone, R.A.; Forgan, R.S.; Fukuyama, H.; Gassensmith, J.J.; Slawin, A.M.Z.; Yaghi, O.M.; Stoddart, J.F. Metal-Organic Framework from Edible Natural Products. Angew. Chem. Int. Ed. 2010, 49, 8630-8634. [CrossRef] [PubMed]

9. Michida, W.; Ezaki, M.; Sakuragi, M.; Guan, G.; Kusakabe, K. Crystal growth of cyclodextrin-based metal-organic framework with inclusion of ferulic acid. Cryst. Res. Technol. 2015, 7, 556-559. [CrossRef]

10. Michida, M.; Nagai, A.; Sakemura, T.; Sakuragi, M.; Mizuki, K.; Kusakabe, K. Flourescent properties of flourescein and rhodamine B in cyclodextrin-based metal-organic framework. Kagaku Kogaku Ronbunshu 2018, 44, 1-5. [CrossRef]

11. Brown, D.H.; Smith, W.E. Gold thiolate complexes in vitro and in vivo. ACS Symp. Ser. 1983, 309, 401-418.

12. Guo, Y.; Tong, X.; Ji, L.; Wang, Z.; Wang, H.; Hu, J.; Pei, R. Visual detection of $\mathrm{Ca}^{2+}$ based on aggregation-induced emission of $\mathrm{Au}(\mathrm{I})-\mathrm{Cys}$ complexes with superb selectivity. Chem. Commun. 2015, 51, 596-598. [CrossRef] [PubMed]

Publisher's Note: MDPI stays neutral with regard to jurisdictional claims in published maps and institutional affiliations.

(C) 2020 by the authors. Licensee MDPI, Basel, Switzerland. This article is an open access article distributed under the terms and conditions of the Creative Commons Attribution (CC BY) license (http://creativecommons.org/licenses/by/4.0/). 